\title{
Perfil, motivaciones e intereses de los aprendices mayores hacia los programas universitarios
}

\section{Profile, motivations, and interests of senior learners towards University Programs}

\section{Perfil, motivações e interesses do aluno maior para os programas universitários}

\author{
Sara Serrate González* \\ Ana Belén Navarro Prados** \\ José Manuel Muñoz Rodríguez ${ }^{* * *}$
}

Fecha de recepción: 28 de abril de 2016

Fecha de evaluación: 15 de marzo de 2017

Fecha de aceptación: 8 de mayo de 2017

Disponible en línea: 18 de mayo de 2017

DOI: org/10/18359/reds.1863

Cómo citar este artículo:

Serrate González, S., Navarro Prados, A. B. y Muñoz Rodríguez, J. M. (2017). Perfil, motivaciones e intereses de los aprendices mayores hacia los Programas Universitarios. Revista Educación y Desarrollo Social, 11(1), 156-171. DOI: org/10/18359/reds.1863

Doctora en Educación. Departamento de Teoría e Historia de la Educación, Universidad de Salamanca, España. Correo electrónico: sarasg@usal.es

** Doctora en Psicología. Departamento de Psicología Evolutiva y de la Educación. Universidad de Salamanca, Zamora. España. Correo electrónico: anavarro@usal.es

*** Doctor en Pedagogía. Departamento de Teoría e Historia de la Educación. Universidad de Salamanca, España..Correo electrónico: pepema@usal.es 


\title{
Resumen
}

La educación en la vejez constituye un modo de envejecimiento activo, siendo los programas universitarios para mayores un escenario idóneo para que el alumnado adulto desarrolle nuevas competencias y conocimientos adaptados a sus necesidades. En este estudio se trata de conocer la existencia de cambios en el perfil de los alumnos que participan en el Programa Interuniversitario de la Experiencia de la Universidad de Salamanca y de examinar los motivos e intereses que los han impulsado a matricularse. Se realizó, además, un estudio descriptivo de desarrollo de tendencia (Mateo, 2009). El instrumento aplicado englobaba preguntas organizadas en dos dimensiones (perfil estudiantil y motivaciones de participación). Posteriormente, se examinaron las respuestas obtenidas y se valoraron la frecuencia y el porcentaje de respuesta en las opciones ofrecidas para cada variable estudiada, diferenciando por año y sede del programa, así como el número total de matrícula por curso, año académico y sede del programa. Se encontró que los rasgos que definen el rostro del aprendiz mayor están cambiando, como también sus motivaciones a la hora de ser participantes activos en programas formativos. No podemos hablar de un solo perfil del estudiante universitario mayor. Para dar respuesta a todas estas características diversas y complejas es fundamental diseñar programas basados en metodologías que promuevan nuevos modos de desarrollo personal con un mayor protagonismo en su aprendizaje, aprovechando los recursos y capitales materiales y humanos con los que cuentan las facultades y los centros propios universitarios.

Palabras clave: gerontología educativa, programas universitarios para mayores, envejecimiento activo, mayores.

\begin{abstract}
Education in old age is a way of Active Ageing, with the University Programs for seniors an ideal setting for adult students to develop new skills and knowledge adapted to their needs. The aim of this study is to know the existence of changes in the profile of students participating in the Inter-University Program Experience at the University of Salamanca and examine the motives and interests that are pushing them to enroll, A descriptive study of trend development was performed (Mateo, 2009). The instrument applied encompassed questions organized in two dimensions (student profile and motivations of participation). Subsequently, the answers obtained were examined, and the frequency and percentage of response in the options offered for each variable studied were evaluated, differentiating per year and place of the program, as well as the total number of enrollments per course, academic year and program headquarters. It was found that the traits that define the face of greater learner are changing, as well as their motivations for being active participants in training programs. We cannot speak of a single profile senior college student. To respond to all these diverse and complex characteristics is essential to design programs based on methodologies that promote new ways of personal development with a greater role in their learning programs, taking advantage of the material and human resources that are at the faculties and the university centers.
\end{abstract}

Keywords: Educational Gerontology, University programs for seniors, active aging, seniors.

\section{Resumo}

A educação na velhice constitui uma forma de envelhecimento ativo, sendo os programas universitários para idosos um cenário ideal para que os estudantes adultos desenvolvam novas habilidades e conhecimentos adaptados a suas necessidades. Neste estudo procura-se conhecer a existência de mudanças no perfil dos alunos que participam no Programa de Interuniversitário da Experiência, da Universidade de Salamanca e de examinar os motivos e interesses que os têm impulsionado a inscrição. Realizou-se, além disso, um estudo descritivo de desenvolvimento de tendência. O instrumento aplicado abrangia perguntas organizadas em duas dimensões (perfil do estudante e motivações para a participação). Posteriormente, as respostas obtidas foram examinadas e se avaliaram a frequência e a percentagem de resposta nas opções oferecidas para cada variável estudada, diferenciando por ano e sede do programa, assim como o número total de matrículas por curso, ano letivo e sede do programa. Encontrou-se que as características que definem a face do aluno maior estão mudando, como as suas motivações por serem participantes ativos em programas de formativos. Para dar resposta a todas essas características diversas e complexas é essencial projetar programas com base em metodologias que promovam novas formas de desenvolvimento pessoal, com um maior protagonismo em sua aprendizagem, aproveitando os recursos e capitais materiais e humanos que tem as faculdades e os centros próprios universitários.

Palavras-chave: gerontologia educativa, programas universitários para idosos, envelhecimento ativo. 


\section{Introducción}

La educación a lo largo de la vida supone un marco referencial para transformar y emancipar individuos y colectivos, basado en la decisión asumida por personas adultas para emprender procesos formativos, con el objetivo fundamental de conseguir mayor capacidad de acción y bienestar social y personal (Prestes y Diniz, 2015). Concretamente en la etapa de la vejez, la educación repercute en diferentes beneficios para este colectivo, como puede ser, entre otros, el desarrollo de nuevas competencias y conocimientos que les permitan funcionar de manera más autónoma en la vida cotidiana, la mejora del funcionamiento general, el aumento del bienestar personal, la creación de nuevos lazos sociales o la posibilidad de disponer de herramientas para participar y contribuir activamente en ese cambio (Bermejo, 2010; Lucas et al., 2014; Serdio, 2008; Villar, 2005).

La educación en la vejez constituye un modo de envejecimiento activo, concepto descrito como la visión más integrativa de la prevención de la dependencia, puesto que reaplica tanto a personas individualmente, como a los grupos de población. El término activo ha de entenderse de manera integral y en el marco de sus posibilidades máximas, comprendiendo por tanto que activas pueden estar las personas enfermas, las que viven alguna situación de diversidad

158 funcional y las que están retiradas de las ocupaciones del trabajo (Organización Mundial de la Salud, 2011). Pretende, además, ser un refuerzo permanente de la salud en su expresión más integral, como "el bienestar físico, mental y social y no sólo la ausencia de enfermedad", enfatizando en la participación y contribución de los mayores en la sociedad (Bermejo, 2010; Fernández, 2009).

Conocer las circunstancias suscritas al colectivo de aprendices mayores, como son sus características psicológicas, la forma como aprenden, sus motivaciones y actitudes hacia el aprendizaje, etc., puede ser fundamental en la planificación y puesta en práctica de programas educativos adaptados y eficientes. Se habla de características cognitivas, motivacionales y experienciales (Glendenning, 2000; Villar, 2004) como posibilidades educativas en la etapa adulta y en la vejez, que se convierten en la actualidad en un campo de estudio, investigación y reflexión pedagógica (Serdio, 2015).

Existen evidencias de que a medida que nos hacemos mayores empiezan a aparecer declives y deterioros en diversas funciones cognitivas, sensoriales y motoras (Browning, 1995; Cuenca, 2011; Glass, 1996). Sin embargo, quedarnos únicamente con los déficits y limitaciones a medida que avanza la edad sería presentar una visión muy limitada de cómo son las personas mayores y de las posibilidades de enseñanza y aprendizaje que presentan y, por lo tanto, una forma de exclusión del proceso de formación permanente o educación a lo largo de la vida.

El declive asociado a la edad debe ser tenido en cuenta en entornos de aprendizaje a la hora de programar y diseñar 
aspectos organizativos en cuanto a los espacios donde se realicen los procesos de enseñanza y aprendizaje que deben de contar, por ejemplo, con iluminación y condiciones acústicas adecuadas. Del mismo modo, deben tenerse en consideración estrategias metodológicas del docente adaptadas a las características del colectivo adulto, como elaborar materiales legibles y claros, sesiones no superiores a una hora y media, disponer de presentaciones verbales y escritas, tratar de unir los conocimientos nuevos con los previos en cada sesión y materia, etc.

La motivación constituye una de las claves que explican la conducta humana, por lo que estar motivados para aprender ayudará a explicar gran parte de la conducta del aprendiz. Pero, ¿cómo es la motivación en el aprendiz mayor? Los aprendices mayores están altamente motivados y predispuestos al aprendizaje, se tienen objetivos más a corto plazo (Cuenca, 2011).

Para ellos aprender es un fin en sí mismo, pero debe responder a sus necesidades personales, sociales y educativas, debe ser personalizado, cooperativo y colaborativo. Los aprendices mayores no se matriculan en un programa de extensión universitaria, como es evidente, por conseguir un empleo o promocionar laboralmente, sino para aprender contenidos funcionales que les permitan sentirse activos e integrados en una sociedad que avanza y cambia de manera permanente. Cuando aprenden quieren hacerlo en un clima positivo, buscan entender el momento vital que atraviesan y comprender mejor la socie- dad en la que viven. Les gusta aprender de forma activa y globalizada, participar, debatir, etc., sintiéndose los protagonistas en su proyecto de realización personal y social y no solamente meros receptores de información (Cuenca y De Juanas, 2011; Montoro, Pinazo, Pinazo y Tortosa, 2007; Navarrete, 2009). Diversas investigaciones han demostrado que los estudiantes mayores estiman más la participación y la interacción con las personas que los contenidos teóricos y prefieren realizar ejercicios sobre temas cotidianos (Lirio, Alonso y Herranz, 2010).

Entre las razones que mueven a los adultos mayores a participar en actividades educativas se encuentran:

1) Acontecimiento vital que cambia el curso habitual de vida. Por ejemplo, la jubilación, viudedad, síndrome del nido vacío, etc., siendo los dos primeros, los principales motivos para apuntarse a un curso educativo. Asistir a los programas universitarios para mayores les permiten adaptarse mejor a estos cambios vitales (Bishop y Martin, 2007; Villar, 2005).

2) Llenar el tiempo y estar activo. Es decir, sustituyen actividades que realizaban en el pasado por otras que les gustan, con el único fin de mantenerse mental y socialmente activos en todas las esferas de la sociedad (Fernández, García y Pérez, 2014).

3) Función terapéutica de la asistencia a los talleres. Que les permita distanciarse y desconectar de alguna situación problemática que estén viviendo. 
4) Elemento compensador de pérdidas. La asistencia a programas universitarios para mayores les permite adquirir conocimientos que en el pasado no pudieron por circunstancias personales, los deja "reciclarse", etc.

Otro aspecto importante y que marca diferencias en la educación en la vejez es la experiencia vital del aprendiz. Esta actúa como un "filtro" por el que pasan las nuevas vivencias y aprendizajes (Villar y Solé, 2006). Actualmente, no se tiene información suficiente sobre cómo se realiza el proceso de aprendizaje de la persona mayor, pero lo que sí está claro es que debe partir de la experiencia personal, de la realidad cercana, así como de valores, actitudes, conocimientos, posibilidades, etc., aspectos que han ido configurando su propia personalidad y que les permiten afrontar los cambios que vayan aconteciendo en su vida (Merriam y Mohamad, 2000). Por eso, todos los programas educativos con personas mayores han de establecer, como punto de partida, la conexión de los nuevos conocimientos con el bagaje acumulado a lo largo de los años, siendo la motivación y el interés los principales incentivos del aprendiz mayor (Serdio, Díaz y Cifuentes, 2014). La realidad que viven las personas mayores es la que hace que aprendan de un modo específico y es a partir de esa realidad 160 desde donde deben diseñarse los programas formativos, y desarrollar así un tipo de educación diferente para esta etapa de la vida.

\section{El Programa Interuniversitario de la Experiencia}

La universidad, desde hace ya algunas décadas, tiene una importante misión en la extensión de los programas formativos a las personas adultas; los programas universitarios de mayores (en adelante, PUM) son una muestra de ello. Entre sus objetivos está contribuir en la promoción de un envejecimiento activo impulsando la autonomía personal y la prevención de la dependencia; de esta manera, se fomenta la creación de una sociedad para todas las edades (Fernández, García y Pérez, 2014; Serdio, 2015) a través de la expansión cultural en la actual sociedad del conocimiento y de la información que implica la rápida difusión y apertura del saber (García, 2009).

El Programa Interuniversitario de la Experiencia, desarrollado por la Universidad de Salamanca durante los últimos años, se enmarca en una propuesta de nivel autonómica, se desarrolla desde los mismos objetivos y sigue la misma estructura en las diferentes universidades de Castilla y León. El programa al que se hace referencia, dependiente de la Universidad de Salamanca, abarca siete sedes, tres de ellas capitales de provincia y cuatro de ámbito rural, y se dirige en total a más de mil alumnos cada curso académico.

La organización curricular del programa abarca materias obligatorias y optativas de disciplinas diversas, que se realizan durante tres cursos académicos, un total de seis asignaturas por curso. Además, 
el estudiante mayor realiza una serie de horas complementarias asistiendo a conferencias o talleres. Adaptarnos a los nuevos tiempos implica que, una vez finalizados los tres años, para aquellos alumnos que desean continuar en el programa, en torno al 50\% cada año, se realiza una oferta formativa diferente, basada en la especialización en función de intereses, por lo que se puede elegir realizar un curso monográfico en torno a una temática concreta (Patrimonio Histórico, Neurociencias, Gótico, Románico, etc.).

En este contexto, se plantean como objetivos centrales del presente trabajo conocer en qué aspectos ha ido variando el perfil de los alumnos que participan en el Programa Interuniversitario de la Experiencia, examinar los motivos e intereses que les vienen impulsando a matricularse y elaborar propuestas futuras de mejora.

\section{Diseño metodológico}

Para describir el cambio producido en los últimos años en el perfil y las motivaciones de los estudiantes mayores en el programa universitario de la experiencia, se ha realizado un estudio descriptivo de desarrollo de tendencia (Mateo, 2009), que permite medir los cambios en las variables estudiadas a partir de la extracción de muestras diferentes de sujetos en cada punto temporal de interés para el estudio; en este caso, los alumnos que se matriculan en el primer curso del programa cada año. Esta metodología ha permitido sacar conclusiones de estimable valor y predecir los cambios acaecidos y las repercusiones que esos cambios ocasionan a la organización, gestión y dirección de este tipo de iniciativas formativas.

El instrumento de medida utilizado es un cuestionario que aglutina las diferentes variables de estudio de respuesta con opción múltiple, organizadas en dos dimensiones: por una parte, el perfil del estudiante (edad, nivel de estudios, sexo y situación laboral); por otra parte, las motivaciones. También se han tomado como referencia los datos totales de matrícula para valorar el aumento o descenso de la demanda durante los últimos seis cursos escolares.

Para el análisis de los resultados, se han examinado las respuestas obtenidas, valorando la frecuencia y el porcentaje de respuesta en las opciones ofrecidas para cada variable estudiada, y diferenciando por año y sede del programa, así como el número total de matrícula por curso, año académico y sede del programa. Para el análisis estadístico de los datos se ha utilizado el programa SPSS v.21.

\section{Resultados}

El número total de matriculados en el primer curso de la Universidad de la Experiencia no supone una cifra constante, como puede observarse en la figura 1. Quienes mayores oscilaciones sufren en número de alumnos por años son principalmente las sedes rurales. $\mathrm{Si}$ nos atenemos a los últimos seis años, se 
observa que el curso 2011/12 fue el más deficiente en número de matriculados, lo que coincide con circunstancias derivadas del momento socioeconómico que ocasionaron recortes financieros y falta de apoyo institucional en el programa. Esto repercutió en una reducción del número de horas por materia en sedes como Salamanca y del número de materias en sedes más pequeñas. El programa sufrió las consecuencias de dichas circunstancias al obtener, de forma generalizada, menos matriculados que otros años, datos que se encuentran en consonancia con una reducción del número de alumnos a nivel autonómico, que ocasionó un $5 \%$ de alumnos nuevos menos en todas las universidades en dicho curso académico. Esto redujo hasta un $15 \%$ menos en el curso 2012/13, pasando de 923 estudiantes de primero a 790 en toda Castilla y León. Comenzó un nuevo repunte a partir del curso académico 2012/13, pasando de los 221 matriculados a los 318 con los que contamos en la actualidad en primer curso, lo que supone el $38,78 \%$ del total de estudiantes matriculados en los PUM de nuestra comunidad.

Los destinatarios de los PUM, concretamente en el de la Junta de Castilla y León, son personas mayores de 55 años, y existe la posibilidad de acceso para personas entre 50 y 55 años. Si nos centramos en la variable "edad" de los participantes, se aprecia una clara tendencia a que los destinatarios potenciales del programa cada vez sean las personas de menor edad, pasando del $15,3 \%$ de alumnos entre 50 y 59 años en el curso 2009/10 al 30,9\% durante el curso 2014/15. Por su parte, el colectivo de alumnos que tienen más de 75 años ha pasado de ser de un $9 \%$ a un 1,65\% (ver figura 2).

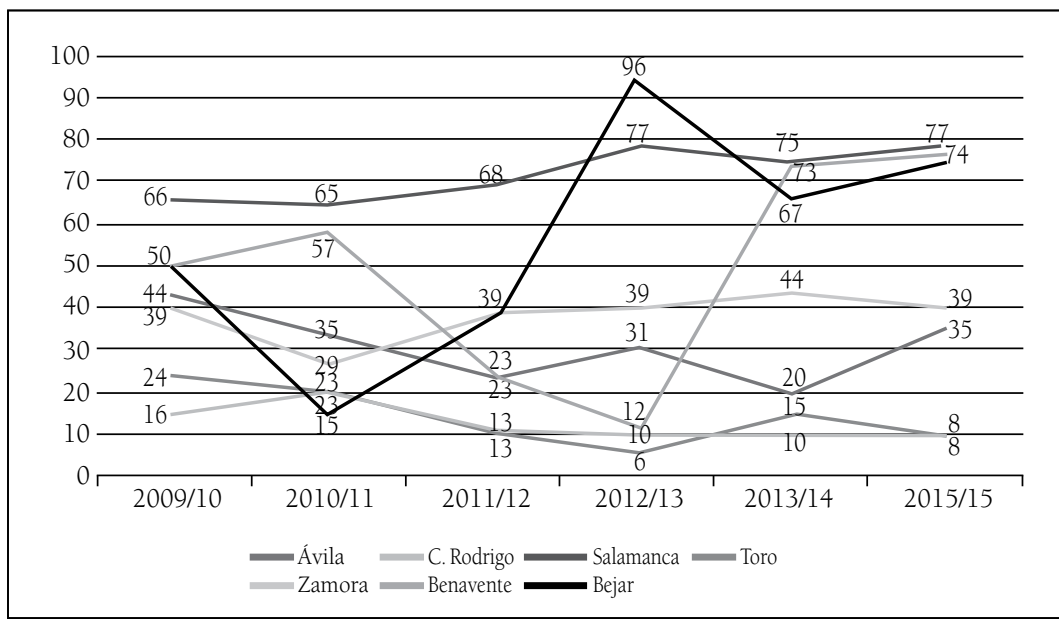

Figura 1. Evolución del número de alumnos en las diferentes sedes del programa

Fuente: elaboración propia. 


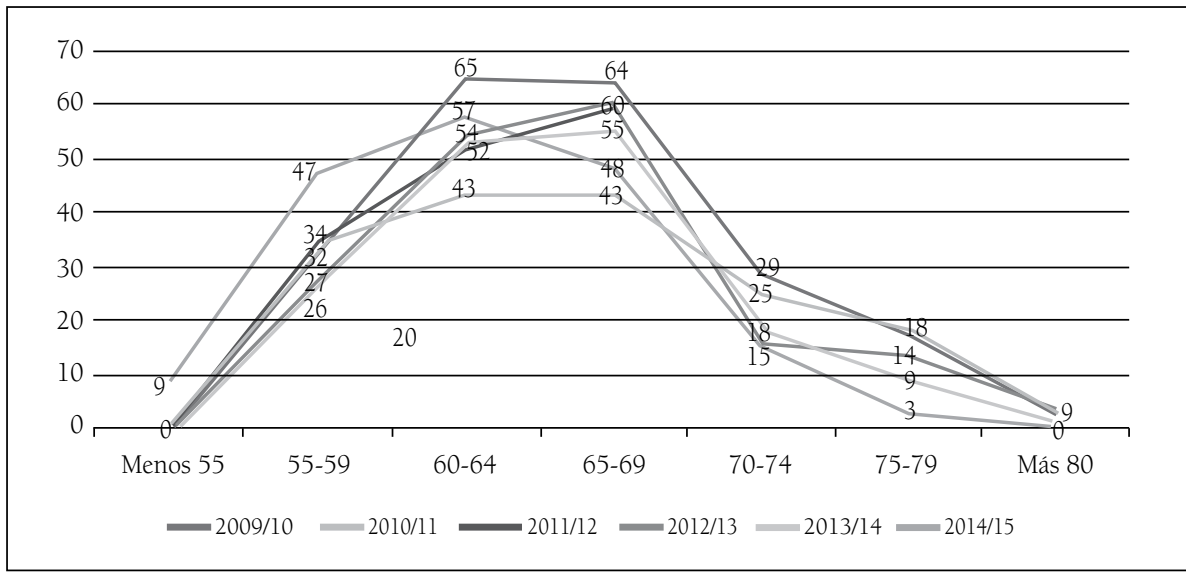

Figura 2. Edad de acceso de los alumnos a lo largo de los últimos seis cursos académicos

Fuente: elaboración propia.

Otro indicativo del nuevo perfil del alumno adulto destinatario de este tipo de programas es el nivel de estudios. En los últimos años asistimos a un cambio generacional asociado a la edad de acceso al Programa, que implica que nos encontremos con alumnos que tienen un mayor nivel de estudios previo. Aun así, se mantiene un claro perfil de personas sin estudios que acceden a la universidad por primera vez, así como personas que han cursado algún tipo de formación profesional. Este colectivo de mayores sin formación permite desmitificar las hipótesis de determinados estudios que avalan que la falta de formación supone un elemento de inmovilidad y pasividad, así como determinante de la baja autoestima y el complejo de inferioridad (Lirio, Alonso y Herranz, 2010). Recibir cada año entre un $10 \%$ y un $15 \%$ de alumnos sin formación previa confirma que los mayores son personas activas con inquietudes educativas que buscan en los programas universitarios una oportunidad de adquirir conocimientos nuevos que les permitan seguir envejeciendo activamente. Esta es la idea de la nueva cultura de la vejez (Serdio, 2015), que se traduce en una vivencia cada vez más sana del envejecimiento, donde se valora la calidad de vida en su máximo exponente a través de la educación.

En la figura 3 se puede observar un descenso en el número de sujetos tan solo con estudios de primaria y bachillerato elemental pasando del 55,71\% del total de alumnos matriculados en primer curso al 35,75\%, aumentando por el contrario el número de alumnos con estudios de bachillerato superior y estudios universitarios, que representan el 43,57\% actualmente frente al 27,61\% en el curso 2009/10.

Estos hechos producen cambios significativos en el perfil de los mayores 
universitarios, que están ocasionados por el incremento producido en el acceso a la educación superior, ligado a un mayor nivel socioeconómico y al desarrollo de políticas de educación encaminadas a la educación en la vejez (García, 2009: Villar, 2005).

Unido al descenso en la edad de los alumnos mayores y al aumento del nivel educativo, está el hecho de que la situación laboral de los participantes tampoco es la misma, y se encuentra un menor número de personas jubiladas, pues pasa del $60,9 \%$ al $42,8 \%$ que representan en la actualidad, y un mayor número de personas en activo o en situación de desempleo, que durante el curso 2009/10 representaban el 7,4\% y en la actualidad suponen el 33,1\% de los alumnos que por primera vez se matriculan en el programa (ver figura 4).

Estos datos están relacionados, entre otros aspectos, con los cambios en la visión social del mayor, con el interés por mantener la actividad intelectual como modo de prevenir el deterioro cognitivo y con su necesidad vital de ocupar el tiempo libre derivado del periodo socioeconómico y cultural del momento (por ejemplo, prejubilaciones o jubilaciones anticipadas, situaciones de desempleo, etc.). En la actualidad, se está presenciando un cambio en el perfil de las personas adultas-mayores, pues hasta hace poco una persona que se jubilaba pasaba de estar activa a inactiva; sin embargo, actualmente necesitan de un grado más alto de participación y protagonismo social y los PUM son el escenario idóneo para conseguir ese empoderamiento (Fernández, García y Pérez, 2014; Iacub y Arias, 2011; Serdio, Díaz y Cifuentes, 2014).

Respecto al sexo de los participantes, no se encuentran cambios significativos en los últimos seis cursos académicos, $\mathrm{y}$ de forma general en todas las sedes donde se imparte el programa se observa que son más el número de mujeres, alrededor del $70 \%$, que el de hombres, que oscila entre el $28 \%$ y el $32 \%$ en la actualidad. Esta feminización del programa es similar a otros PUM a nivel nacional.

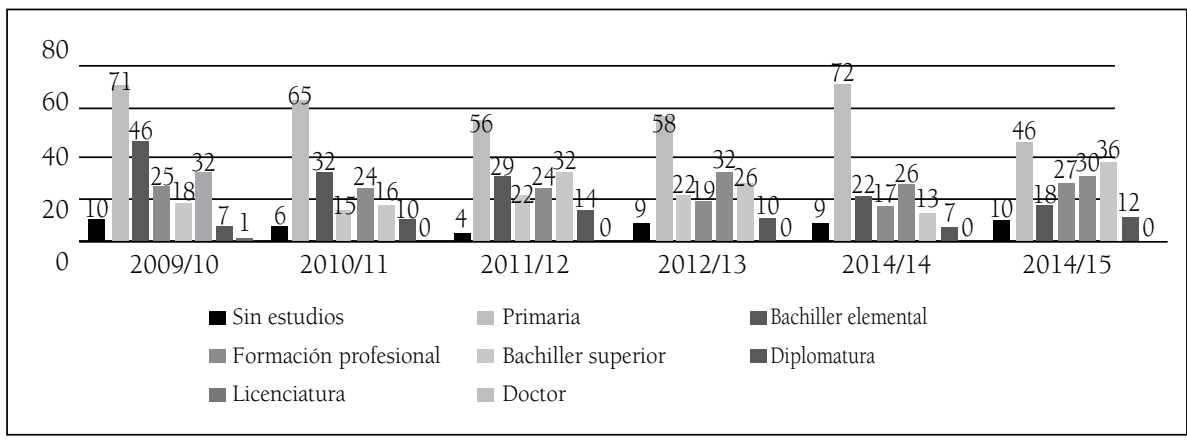

Figura 3. Nivel educativo de los estudiantes mayores

Fuente: elaboración propia. 


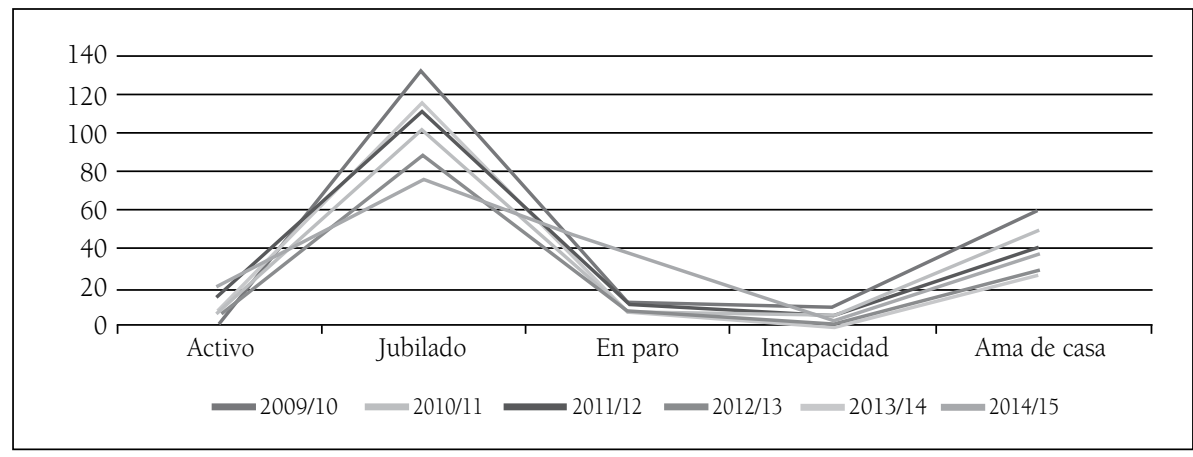

Figura 4. Situación laboral de los aprendices mayores

Fuente: elaboración propia.

En último lugar, cuestionarse los motivos por los que las personas mayores deciden ser partícipes de un programa universitario es importante, en aras de articular proyectos y programas eficaces y de calidad. En este sentido, los alumnos del Programa Interuniversitario de la Experiencia acuden a este motivados por aspectos relacionados, fundamentalmente, con el interés por aumentar o recordar los conocimientos ya adquiridos en otros momentos vitales, mantenerse activos y relacionarse con otras personas. Estas mismas motivaciones se mantienen en el tiempo, como puede observar en la figura 5, y coinciden plenamente con los objetivos que intrínsecamente persigue el programa, como facilitar el acercamiento de las personas mayores al conocimiento, promover espacios de participación e intercambio de relaciones y favorecer el aprendizaje permanente y el crecimiento personal que repercutan en un envejecimiento activo positivo.

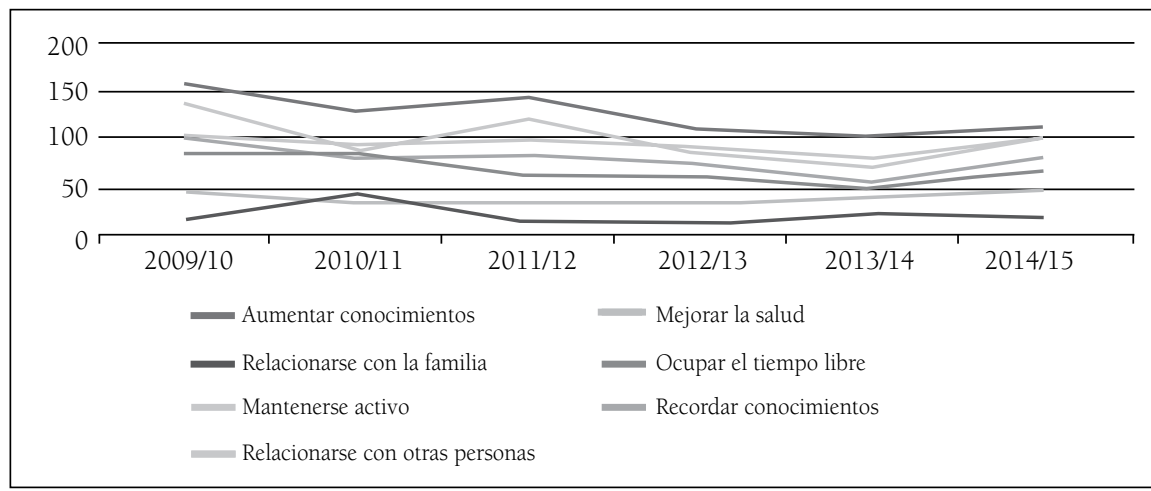

Figura 5. Motivaciones de los estudiantes mayores para participar en el programa

Fuente: elaboración propia. 


\section{Perspectiva de futuro y propuestas de mejora}

Situándonos en la evolución a lo largo de los últimos años, los rasgos que definen el rostro del aprendiz mayor están cambiando, como también sus motivaciones a la hora de ser participantes activos en programas formativos. El perfil del participante mayor en los programas universitarios ha ido variando, hasta configurarse con rasgos propios, adaptados a un contexto social actual en continuo cambio y transformación, y así se da respuesta y se ajusta a los requerimientos sociales y culturales.

Teniendo en cuenta todos los datos referenciados, no podemos hablar de un solo perfil del estudiante universitario mayor, sino de muchos y muy variados. Para dar respuesta a todas estas características diversas y complejas es fundamental diseñar programas basados en metodologías más constructivistas que no se limiten a compensar declives y limitaciones de los mayores, sino que promuevan nuevos modos de desarrollo personal con un mayor protagonismo en su aprendizaje, y se alejen así de métodos unidireccionales donde el profesor es el único transmisor de conocimiento y no permite un papel activo de los aprendices mayores para mostrar todas sus potencialidades (Alfageme, 2009; Arnay, Marrero y Fernández, 2011; Padilla et al., 2012; Serdio, 2015).

Conocer, desde una perspectiva sociohistórica, cómo ha ido variando el perfil del alumnado adulto participante de programas universitarios y concretar su actual caracterización favorece la planificación de este tipo de iniciativas y la adaptación a las nuevas necesidades de los destinatarios en un escenario propicio de aprendizaje en la vejez, como es el espacio universitario.

Abordar programas de educación de adultos cuyos alumnos cada vez se encuentran más formados y preparados, con mayor ocupación de su tiempo libre, que en ocasiones compaginan con su desempeño profesional, obliga a emprender acciones que favorezcan su correcta participación activa en la sociedad, en una sociedad en constante cambio, que obliga a las personas a poner en práctica habilidades adaptativas si no quieren sufrir un aislamiento social que lleve, por lo tanto, a un aislamiento personal. Además, para dichas personas la educación tiene un papel relevante en su bienestar y calidad de vida (Amorós et al., 2006).

Para dar respuesta a las expectativas e inquietudes del nuevo adulto o mayor universitario deben diseñarse y ponerse en práctica procesos de innovación docente que se adapten a las nuevas posibilidades de enseñanza-aprendizaje, y fomentar de esta manera una práctica educativa en la vejez con contenidos, metodologías y estrategias más diversificadas y activas. Nos referimos al desarrollo de habilidades relacionadas con el aprendizaje de idiomas, el uso y manejo de las nuevas tecnologías, el aprendizaje de nuevas formas de comunicación a través de las redes sociales (Blázquez y Holgado, 2011; Lorente, 2011; Villar, 2001; Villar y Solé, 
2006), así como el recurrente aspecto de la salud y el ejercicio físico. A través de ellas los aprendices mayores deben obtener beneficios sociales, emocionales, psicológicos, culturales, de salud, etc., aspecto que favorece que las ganas y el interés por aprender se mantengan en el tiempo. Es importante también, en un marco como el universitario, fomentar en los alumnos adultos y mayores el interés por la ciencia y los centros de investigación, a través de un conocimiento desde los propios espacios donde se genera el conocimiento científico, aprovechando los recursos y capitales materiales y humanos con los que cuentan las facultades y centros propios universitarios (Serrate et al., 2016).

Si el perfil de las personas adultas y mayores que participan en los programas universitarios está cambiando y nos encontramos con personas más formadas y preparadas competencialmente, es importante involucrarles en el proceso de enseñanza-aprendizaje, fomentar el rol activo del estudiante y procurar metodologías que se distancien de la docencia magistral y procuren espacios de reflexión e intercambio en los que el alumnado pueda, teniendo en cuenta su experiencia acumulada, seguir formándose y acumular conocimiento en un proceso educativo permanente y activo a lo largo de su vida. Deben ser los propios destinatarios quienes generen conocimiento, lo difundan y participen de los aprendizajes autodirigidos (Causapié et al., 2011) y en este sentido hacer uso de su experiencia puede favorecer el aprendizaje de generaciones más jóvenes, compartiendo con ellas el acceso al aprendizaje (Blázquez y Holgado, 2011; Macías et al., 2011; Sánchez, 2007; Serrate et al., 2016) para convertirse así en modelos óptimos de envejecimiento.

Los estudios que se han realizado, cuyo foco de atención son los alumnos universitarios adultos y mayores, centran su atención en aspectos como la evaluación de su impacto en la autopercepción del apoyo social y la salud mental (Barceló et al., 2015; Lucas et al., 2014), en la compensación, la reserva cognitiva y el potencial de aprendizaje como formas adaptativas de frenar el deterioro o en los niveles de bienestar psicológico, el autoconcepto y su percepción respecto a la calidad de vida (Padilla et al., 2012).

Todos estos programas basan su eficacia en un conocimiento amplio de los aprendices mayores, y les aportan saberes, competencias, estrategias y apoyos que les sirvan para mejorar o minimizar los efectos negativos del envejecimiento en su bienestar y calidad de vida. Asimismo, consideran fundamental que los profesionales de la educación en la vejez cuenten con la capacidad de crear programas y actividades innovadoras dentro de un contexto donde los futuros estudiantes mayores tienen una responsabilidad más grande hacia el cuidado de su propia salud, más nivel educativo y más participación en la realidad social (Lawton, Moss, Winter, Hoffman, 2007; Serdio, 2008). 
Para adaptar los planes de estudio de los PUM, se deben buscar nuevas fórmulas de actuación educativa conforme a nuevos planteamientos y objetivos (Serdio, 2015). Una de las metas prioritarias es llegar al mayor número de destinatarios que puedan estar interesados en participar en este tipo de iniciativas. Es por ello que existen experiencias puestas en práctica de universidades para mayores en formato on-line o a distancia o cursos semipresenciales que complementan los programas presenciales (Bru, 2010). No obstante, este tipo de iniciativas deben suponer un aumento de los recursos y no una sustitución de los programas universitarios tradicionales que siguen favoreciendo uno de los objetivos concretos que conecta con las motivaciones del alumnado adulto, concerniente al fomento de las relaciones interpersonales.

Sin dejar de lado metodologías tradicionales, se pueden empezar a utilizar metodologías activas de aprendizaje que hasta ahora se han usado con poblaciones jóvenes, adaptándolas a este grupo de individuos (Bermejo, 2005). Por ejemplo, el método del aprendizaje basado en problemas puede aportar en los estudiantes mayores una oportunidad de adquirir competencias clave a partir del trabajo cooperativo (Soria, 2012). Existen pocos estudios que muestren resultados utilizando metodologías más activas con población mayor (Anguloy Cuenca, 2011;

168 De Vicente et al., 2011). Por este motivo, desde el Programa Interuniversitario de la Experiencia se están empezando a desarrollar propuestas formativas en esta línea, con el objetivo de ayudar en su empoderamiento. De la misma forma, el recurso intergeneracional ha dado y sigue dando buenos resultados de aprendizaje, las jornadas en las que se realiza un trabajo conjunto alumnos jóvenes con alumnos mayores, pero también experiencias de aprendizaje-servicio, en las cuales estudiantes jóvenes, preprofesionales, a la vez que continúan su proceso de aprendizaje, prestan un servicio al programa desarrollando un taller formativo con alumnos adultos. Las relaciones que establecen entre ellos, así como los resultados del aprendizaje, reportan beneficios a unos y a otros (Serrate et al., 2016).

No obstante, consideramos que en un intento de adaptar la universidad y los programas universitarios a los nuevos requerimientos sociales, es imprescindible realizar un diagnóstico completo de esta población que lleve a conocer y concretar en mayor medida las motivaciones y expectativas que orientan su aprendizaje para ofertar una verdadera educación en la vejez, donde se dé respuesta a las demandas socioeducativas de este grupo cada vez más heterogéneo e implicado en programar su prejubilación y jubilación con multitud de proyectos, intenciones y actividades (Valle Aparicio, 2014).

Los PUM deben sustentarse en un nuevo modelo de desarrollo de la vejez, que entienda esta etapa como un momento de la vida lleno de posibilidades, en los que la actividad y la participación se conviertan en la base del desarrollo personal para atravesar la vejez con energía (Padilla et al., 2012). 


\section{Referencias}

Alfageme, A. (2009). El retiro temporal a lo largo del curso vital: contexto, regulación y consecuencias. Recerca, 9, 41-58.

Amorós, P., Bartolomé, M., Sabariego, M. y De Santos, J. (2006). Construyendo futuro. Las personas mayores: una fuerza social emergente. Madrid: Alianza.

Angulo, A. y Cuenca, N. (2011). Participación colaborativa de alumnos mayores en prácticas experimentales de laboratorio y en casos de aprendizaje basado en problemas de asignaturas de Ciencias de la Salud. En C. Bru Ronda (Coord.), Aprendizaje a lo largo de la vida, envejecimiento activo y cooperación internacional en los programas universitarios para mayores. IV Congreso Iberoamericano de Universidades para Mayores. Alicante: AEPUM

Arnay, J., Marrero, J., y Fernández, I. (2011). Las universidades para mayores: ¿qué enseñanza, qué aprendizaje?. Revista de Ciencias de la Educación, 225-226, 89-106.

Barceló, M., Orte, C., y Ballester, L. (2015). Efectos de los programas universitarios en personas mayores en su red y apoyo social. El ejemplo de la Universitat Oberta Per a Majors. Pedagogía Social. Revista Interuniversitaria, 25, 299-317.

Bermejo, L. (2005). Gerontología Educativa. Cómo diseñar proyectos educativos para personas mayores. Madrid: Editorial Médica Panamericana.

Bermejo, L. (2010). Envejecimiento activo y actividades socioeducativas con personas mayores. Madrid: Editorial Médica Panamericana.
Bishop, AJ., y Martin, P. (2007). The indirect influence of educational attainment on loneliness among unmarried older adults. Educational Gerontology, 33, 897-917.

Blázquez, F., y Holgado, A. (2011). Innovación educativa en los programas universitarios para mayores. Revista de Ciencias de la Educación, 225-226, 129-150.

Browning, C.J. (1995). Late-life education and learning: The role of cognitive factors. Educational Gerontology, 21(5), 401-413.

Bru Ronda, C. (2010). La enseñanza universitaria para personas mayores: Evolución conceptual, innovación educativa y adaptación a las demandas sociales del siglo XXI. Investigación y buenas prácticas de trabajo académico con mayores en España. Revista Palabras Mayores, 5, 1-30.

Causapié,P., Balbontín, A., Porras, M. y Mateo, A. (Coords.) (2011). Envejecimiento Activo. Libro Blanco. Madrid: Ministerio de Sanidad, Política Social e Igualdad [Imserso].

Chang, D. y Lin, S. (2011). Motivation to learn among older adults in Taiwan. Educational Gerontology, 37, 574-592.

Cuenca, E. (2011). Motivación hacía el aprendizaje en las personas mayores más allá de los resultados y el rendimiento académico. Revista de Psicología y Educación, 6, 239-254.

Cuenca, E. y De Juanas, A. (2011) Experiencia de aprendizaje cooperativo en un contexto virtual universitario.

En J. M. Román, M. A. Carbonero y J. D. Valdivieso (Comps.), Educación, 
aprendizaje y desarrollo en una sociedad multicultural (pp. 5679-5689).Madrid: Ediciones de la Asociación Nacional de Psicología y Educación.

De Vicente, F. y Mateu, M. J. (2011). Innovación y mejora docente en la impartición de asignaturas jurídicas en las universidades de mayores: una experiencia y propuesta educativa. En C. Bru Ronda (Coord.), Aprendizaje a lo largo de la vida, envejecimiento activo y cooperación internacional en los programas universitarios para mayores. IV Congreso Iberoamericano de Universidades para Mayores. Alicante: Asociación Estatal de Programas Universitarios para Mayores [Aepum].

Fernández, R. (2009). Envejecimiento activo: contribuciones de la psicología. Madrid: Pirámide.

Fernández, A., García, J.L. y Pérez, G. (2014). Los programas universitarios de mayores y su contribución al aprendizaje a lo largo de la vida. Revista Complutense de Educación, 25(2), 521-540.

García, J. (2009). Abriendo nuevos campos educativos. Hacia la educación en personas Mayores. Revista Historia de la Educación Latinoamericana, 12, 129-151.

Glass, J.C. (1996) Factors affecting learning in older adults. Educational Gerontology, 22, 359-372.

Glendenning, F. (2000). Teaching and learning in later life. Theoretical implications. Aldershot: Arena.

Iacub, R. y Arias, C.J. (2011). El empoderamiento en la vejez. Journal of Behavior, Health E Social Issues, 2(2), 25-32.

Lawton, M. P., Moss, M. S., Winter, L. y Hoffman, C. (2007). Motivation in later life: personal projects and wellbeing.
Psychology and aging, 17(4), 539-547.

Lirio, J., Alonso, D. y Herranz, I. (2010). Envejecer participando. El proyecto "Entre mayores". Una experiencia de investigaciónacción. Buenos Aires: Miño y Dávila.

Lorente, X. (2011). Nuevas tecnologías, aprendizaje colaborativo y creatividad en las personas mayores. Revista de Ciencias de la Educación, 225-226, 151-163.

Lucas-Molina, B., Pérez-Álbeniz, A., Fonseca, E. y Ortuño-Sierra, J. (2014). Programas educativos para mayores: evaluación de su impacto en la autopercepción del apoyo social y la salud mental. Revista Colombiana de Psicología, 24(1), 47-60.

Macías, L., Orte, C. y March, M. (2011). Programas universitarios para mayores y relaciones intergeneracionales. La experiencia de un programa. Revista de Ciencias de la Educación, 225-226, 227-250.

Mateo, J. (2009). La investigación ex postfacto. En R. Bisquerra (Ed.), Metodología de la investigación educativa (pp. 195230). Madrid: La Muralla.

Merriam, S. B. y Mohamad, M. (2000). How cultural values shape learning in older adulthood: The case of Malaysia. Adult Education Quarterly, 51(1), 45-63.

Montoro, J., Pinazo, S. y Tortosa, M.A. (2007). Motivaciones y expectativas de los estudiantes mayores de 55 años en los programas universitarios. Revista Española de Geriatría y Gerontología, 15(3), 178-187.

Navarrete, B. (2009). La motivación en el aula. Funciones del profesor para mejorar la motivación en el aprendizaje. Innovación y Experiencias Educativa, 15, $1-9$. 
Organización Mundial de la Salud [OMS] (2011). Libro blanco del envejecimiento activo. Madrid: Imserso.

Padilla-Góngora, D., López-Liria, R., Martínez-Martínez, A., Soro, G. y Pérez, D. (2012). Diseño y experimentación de un programa educativo para mayores en Almería: Proyecto Grundtvig. International Journal of Developmental and Educational Psychology, 1(3), 309-315.

Prestes, E. y Diniz, A. (2015). Educación y aprendizaje a lo largo de la vida: los adultos y la enseñanza superior. Sinéctica, 45, 1-20.

Sánchez, C. L. (2007). La responsabilidad social universitaria en el contexto del cambio de la Educación Superior. Recuperado de http://e-spacio. uned.es/fez/eserv/bibliuned:19308/ n02martinecar07.pdf

Serdio, C. (2015). Educación y envejecimiento: una relación dinámica y en constante transformación. Educación XX1, 18(2), 237-255.

Serdio, C. (2008). La educación en la vejez: fundamentos y retos de futuro. Revista de Educación, (346), 467-483.

Serdio, C., Díaz, B. y Cifuentes, P. (2014). Envejecer activamente, aprender activamente: apuntes para una propuesta educativa en el ámbito universitario. International Journal of Developmental and Educational Psychology, 2(1), 91-98.

Serrate, S., Muñoz, J.M. y Alba, J. (2016). Aprendizaje-servicio en la Universidad de la Experiencia. En Actas XIV Encuentro AEPUM "Nuevos perfiles de alumnos en las Aulas de Mayores. Reflexiones en torno a sus percepciones y expectativas sobre los Programas Universitarios para Mayores en España". Granada: Asociación Estatal de Programas Universitario de Mayores [en prensa].

Soria, V. (2012). El aprendizaje basado en problemas (ABP). Una propuesta metodológica para la formación de adultos/as. Diálogos, 70, 43-50.

Valle Aparicio, J. E. (2014). Educación permanente: los programas universitarios para mayores en España como respuesta a una nueva realidad social. Revista de Educación Superior, 43(171), 117-138.

Villar, F. (2001). ¿Tiene sentido la formación en la vejez? Las nuevas tecnologías como ejemplo paradigmático. Comunicación y Pedagogía, 173, 57-62.

Villar, F. (2004). Educación y personas mayores: algunas claves para la definición de una psicología de la educación en la vejez. Revista Brasileira de Ciencias do Envelhecimiento Humano, 1, 61-76.

Villar, F. (2005). Educación en la vejez: hacia la definición de un nuevo ámbito para la psicología de la educación. Infancia y Aprendizaje, 28(1), 63-79.

Villar, F. y Solé, C. (2006). Intervención psicoeducativa con personas mayores. En C. Triadó y F. Villar (Eds.), Psicologia de la Vejez (pp. 423-450). Madrid: Alianza. 